\title{
Current status and preventive strategies of chronic obstructive pulmonary disease in China: a literature review
}

\author{
Zhenyu Quan ${ }^{1 \#}$, Guanghai Yan ${ }^{2,3 \#}$, Zhiguang Wang ${ }^{2,4}$, Yan $\mathrm{Li}^{2,4}$, Jie Zhang ${ }^{5}$, Ting Yang ${ }^{6}$, Hongmei Piao \\ ${ }^{1}$ Department of Public Health, Yanbian University Medical College, Yanji, China; ${ }^{2}$ Jilin Key Laboratory for Immune and Targeting Research on \\ Common Allergic Diseases, Yanbian University, Yanji, China; ${ }^{3}$ Department of Anatomy, Histology and Embryology, Yanbian University Medical \\ College, Yanji, China; ${ }^{4}$ Department of Respiratory Medicine, Affiliated Hospital of Yanbian University, Yanji, China; ${ }^{5}$ Department of Respiratory and \\ Critical Care Medicine, The Second Hospital of Jilin University, Changchun, China; ${ }^{6}$ Department of Pulmonary and Critical Care Medicine, Center \\ of Respiratory Medicine, China-Japan Friendship Hospital, Beijing, China \\ Contributions: (I) Conception and design: T Yang, H Piao; (II) Administrative support: G Yan, Hongmei Piao; (III) Provision of study materials or \\ patients: J Zhang, Y Li; (IV) Collection and assembly of data: Z Quan, G Yan, Z Wang, Y Li; (V) Data analysis and interpretation: Z Quan, Z Wang, \\ Y Li; (VI) Manuscript writing: All authors; (VII) Final approval of manuscript: All authors. \\ "These authors contributed equally to this work. \\ Correspondence to: Hongmei Piao. Department of Respiratory Medicine, Affiliated Hospital of Yanbian University, No. 1327, Juzi Street, Yanji 133000, \\ China. Email: piaohm1969@126.com; Ting Yang. Department of Pulmonary and Critical Care Medicine, Center of Respiratory Medicine, China- \\ Japan Friendship Hospital, No. 2 Yingyuan East Street, Chaoyang District, Beijing 100029, China. Email: dryangting@qq.com.
}

\begin{abstract}
Chronic obstructive pulmonary disease (COPD) is a common respiratory disease that seriously threatens human health and wellbeing, thereby representing an important public health problem. At present, it is the fourth leading cause of death worldwide, and is estimated to become the third greatest cause of death by 2030. In China, the prevalence of COPD is increasing, secondary to an increase in smoking, air pollution and an aging population, resulting in a current the mortality of COPD in China which is higher than the global average. Moreover, the disability-adjusted life year (DALY) rate of COPD in China is still relatively high, with an associated heavy economic burden to patients, their families and society. Unfortunately, current measures for treatment and prevention of COPD in China are not optimal. This primarily results from limited public awareness of COPD and pulmonary function tests amongst residents of China, and the generally poor disease-specific knowledge of primary care doctors. In recent years, a series of preventative strategies have been introduced in China across at the level of national policy, societies and associations, and scientific research. This review focuses upon both the epidemiology of COPD and the current status of preventative and treatment strategies in China.
\end{abstract}

Keywords: Chronic obstructive pulmonary disease (COPD); epidemiology; prevention strategies

Submitted Jun 02, 2020. Accepted for publication Apr 22, 2021.

doi: 10.21037/jtd-20-2051

View this article at: http://dx.doi.org/10.21037/jtd-20-2051

\section{Prevalence of chronic obstructive pulmonary disease (COPD)}

During the past few decades, the prevalence of COPD in China has increased such that the total prevalence of COPD in China has increased from $2.70 \%$ in 1990 to $3.84 \%$ in 2010. This increase was greater in elderly patients (1990:
$0.49 \%$ in $<20$ years old, $20.95 \%$ in $\geq 80$ years old; 2010 : $0.55 \%$ in $<20$ years old, $22.89 \%$ in $\geq 80$ years old). The total number of patients with COPD increased by $66.73 \%$ from 30 million in 1990 to 50 million in 2010 (1). In 1991, the national prevalence of COPD in people over 15 years of age was 3\% (2). From 2002 to 2004, the results of a survey in seven provinces and cities of China showed that the 
prevalence of COPD was $8.2 \%$ in people aged $\geq 40$ years; $12.4 \%$ in men and $5.1 \%$ in women; $7.8 \%$ in rural areas and $8.8 \%$ in urban areas (3). From 2012 to 2015, a survey in adults aged $\geq 20$ years old found that the prevalence of COPD was $8.6 \%$ in people aged $\geq 20$ years old $(11.9 \%$ in men and $5.4 \%$ in women), and this represented $9.4 \%$ and $7.4 \%$ of the rural and urban population respectively. Moreover, the prevalence of COPD increased with age, with $13.7 \%$ in people aged $\geq 40$ years old and $35.5 \%$ in people aged $\geq 70$ years old (4). From 2014 to 2015, national survey data suggested that the standardized prevalence of COPD was $13.6 \%$ in people aged $\geq 40$ years old $(19.0 \%$ in men and $8.1 \%$ in women), which increased with age (5). However, these data cannot be directly compared due to the use of different epidemiological survey methods, pulmonary function test methods for COPD diagnosis and subject populations.

Overall, the prevalence of COPD in China is still increasing due to the aging of population in China (the population over 60 years old increased from $12.5 \%$ in 2009 to $18.1 \%$ in 2019 ) and the high air pollution and smoking rate (the smoking rate was $26.6 \%$ at the age of 15 years and above). We present the following article in accordance with the Narrative Review reporting checklist (available at http:// dx.doi.org/10.21037/jtd-20-2051).

\section{Risk factors of COPD}

Risk factors for COPD include independent factors such as genetics, causative environmental factors such as smoking, occupational dust exposure and chemical exposure, and indoor/outdoor air pollution. The results of a systematic analysis of studies from 2009 to 2015 showed that the main risk factors for COPD in China were tobacco exposure and the use of biofuels/solid fuels (6).

\section{Smoking}

Numerous studies have confirmed that smoking and second-hand smoke exposure are important risk factors for the morbidity and mortality of COPD. The results of a large study by Fang et al. (5) highlighted that the risk of COPD was 1.87 times (95\% CI: 1.60-2.19) higher amongst smokers relative to non-smokers. Shi et al. (7) reported that the prevalence of COPD in smokers was 2.21 times $(95 \%$ CI: 1.505-3.241) higher than that in non-smokers, and the prevalence of COPD in patients with secondhand smoke exposure was 1.57 times (95\% CI: 1.181-2.097) greater than in non-smokers. The 8-year cohort study by Chen et al. (8) found that COPD mortality of male smokers was 9.09 times (95\% CI: 5.11-16.15) greater than non-smokers. The risk of COPD was 4.11 times (95\% CI: 1.412-3.163) larger in people who smoked more than 20 cigarettes per day than in non-smokers (9). He et al. (10) observed that after 17 years of follow-up the mortality rate of COPD in those exposed to smoking was 2.3 times greater than in the unexposed group (95\% CI: 1.06-5.00).

Unfortunately, there may be 1.9 million non-smokers in China who suffer from COPD due to secondhand smoke exposure, which in turn leads to death (11). Surveillance of chronic diseases and risk factors in China showed that the prevalence of smoking among Chinese men remained at a high level $(58.7 \%, 58.2 \%$, and $54.0 \%$ of adult men aged $18-69$ years in 2004, 2007, and 2010, respectively) (12). The 2010 Global Adult Tobacco Survey: China Report showed that $72.4 \%$ of non-smokers were exposed to secondhand smoke in China (13). In 2014, the prevalence of smoking was $57.6 \%$ in men and $4.0 \%$ in women, and the exposure to secondhand smoke was $44.9 \%$ in the population aged $\geq 40$ years in China $(14,15)$. The prevalence and mortality of COPD in Chinese smokers is at a high level.

There are 300 million smokers in China and 740 million people are exposed to secondhand smoke (http://www.gov. cn/jrzg/2014-01/07/content_2561314.htm). Therefore, it is important to strengthen tobacco control legislation, prohibit smoking in public places and publicize tobacco hazards especially for adolescents.

The "Notice on Further Strengthening Smoke Control among Adolescents" jointly issued by the Health and Reform Commission and the Ministry of Education included the following proposals. First, knowledge related to tobacco control should be included in the health education courses of primary and secondary schools. Secondly, public health education should be carried out widely to facilitate a public understanding of the health hazards resulting from secondhand smoke and thereby improve public rejection of secondhand smoke exposure, alongside active participation in tobacco control activities. Finally, public education will support progress towards the proposed smoking rate of $20.0 \%$ among people aged 15 years and above proposed as in the Outline of the 2030 Plan for Healthy China.

\section{Pollution from fuel smoke}

Household indoor air pollution from contaminated fuel combustion is an important public health problem in 
China and even worldwide. Pollution causing fuels include biofuels, coal, kerosene, and paraffin, which can release a variety of harmful substances such as $\mathrm{SO}_{2}, \mathrm{NO}_{x}$, and fine particulate matter (PM2.5; $<2.5 \mu \mathrm{m})$. Burning these fuels for cooking or heating in indoor areas with poor ventilation can cause an increase in harmful indoor pollutants, which is the main factor leading to COPD. Among Chinese nonsmoking women, the incidence of COPD in rural women was 3.60 times (95\% CI: $1.13-11.52$ ) higher than that in urban women (16), mainly due to the heavy use of organic fuels in rural areas. Ding et al. (9) demonstrated that the risk of COPD was 8.39 times (95\% CI: 4.91-14.66) higher in those exposed to biofuel smoke than in those not exposed. Lin et al. (17) performed a meta-analysis of risk factors associated with COPD in the Chinese population and showed that the risk of COPD was 3.10 times (95\% CI: 1.77-3.71) greater in those who used coal and firewood fuels than in those who were not exposed. A meta-analysis published by An et al. (18) investigated the relationship between biofuel smoke exposure and COPD in a Chinese cohort from January 1990 to December 2015. The authors found that the risk of COPD in biofuel exposed individuals was 1.94 times (95\% CI: 1.49-2.153) and 2.66 times (95\% CI: 1.75-4.04) greater in cross-sectional and case-control studies, respectively. In 2014, COPD monitoring in China demonstrated that the use of contaminated fuels accounted for $59.9 \%$ of fuel usage for home cooking or heating in the population aged 40 years and above higher, which was greater than the global average of $43 \%$ in 2014 (19). Subjects with exposure to contaminated fuels in the female population aged 40 years and above accounted for $44.5 \%$ (20). In summary, biofuel exposure levels are high in Chinese women. Measures should be taken to reduce the exposure to contaminated fuels such as biofuels and coal, thus reducing the risk of COPD.

\section{Occupational exposure}

Occupational exposure is a risk factor for COPD and includes occupational exposure to organic and inorganic dust, chemicals, and Smoke exposure resulting from work in agriculture, mining or heavy industry. The impact of occupational exposure on COPD is often underestimated. Wang et al. (21) reported that in China the exposure to dust and/or harmful gases in the population aged 40 years and above was $46.3 \%$ (95\% CI: $41.7-50.8 \%$ ). The exposure rate was higher in rural areas $(51.7 \%)$ than in towns $(40.3 \%)$, and was higher in males (51.4\%) than in females (41.0\%).
However, the proportion of the exposed population taking protective measures accounted for only $26.7 \%$ of the exposed population. Zhou et al. (22) conducted a study in the population aged 40 years and above in 7 provinces and cities of China and found that $20.5 \%$ of the population were exposed to occupational dust and smoke, and the risk of COPD in the occupationally exposed group was 1.22 times of that in the unexposed group (95\% CI: 1.09-1.38). The risk of COPD was positively correlated with the years of exposure and had a multiplicative interaction with smoking [smoking and occupational exposure odds ratio (OR) $=2.50]$. The results of a study on the influencing factors of COPD conducted in three surveillance sites in Xinjiang in 2015 highlighted that the risk of COPD in people with occupational dust exposure for longer than a 1-year period was 1.43 times of that in non-exposed people (95\% CI: $1.03-1.98)(23)$. Therefore, it is necessary to strengthen the management of safety protection measures (such as wearing protective masks) for occupationally exposed people and ensure the integrity of ventilation equipment in the workplace, reducing the risk of COPD in occupationally exposed people.

\section{Air pollution}

With the extensive development of industry, the large increase of coal energy consumption and the increasing number of automobiles, outdoor air pollutants are also increasing in China. Air pollution in China mainly derives from the exhaust gas emitted by motor vehicles and from building dust. Following an increase in the concentration of larger particulate matter (PM10; $<10 \mu \mathrm{m}), \mathrm{SO}_{2}$ and $\mathrm{NO}_{2}$ by $10 \mu \mathrm{g} / \mathrm{m}^{3}$, the incidence of slow obstructive pulmonary death increased significantly, and the corresponding OR value and $90 \%$ CI were greater than one (24). The strongest cumulative lag effect on respiratory disease deaths and slow obstructive pulmonary disease deaths was attributed to PM2.5, with excess risks of $3.96 \%$ (95\% CI: $0.82-7.19 \%$ ) and $6.12 \%$ (95\% CI: $1.44-11.02 \%$ ) for each increase of $155 \mu \mathrm{g} / \mathrm{m}^{3}$, respectively (25). Jin et al. (26) showed that for each unit $\log$ increase in $\mathrm{SO}_{2}$, total suspended particulate (TSP), PM10, and PM2.5 concentration, the risk of COPD increased by 1.31-, 1.53-, 1.51-, and 1.68-fold, respectively. Additionally, these authors demonstrated that the risk of adult respiratory symptoms and COPD in severely polluted areas were 1.7 and 1.5 times greater than in relatively clean areas (27). Air pollution can significantly increase the rate of outpatient visits and admissions in 
patients with COPD. Li et al. (28) reported in a metaanalysis of $1,128,241$ cases that for every $10 \mu \mathrm{g} / \mathrm{m}^{3}$ increase in atmospheric PM2.5, $\mathrm{PM} 10, \mathrm{SO}_{2}, \mathrm{NO}_{2}$ and $\mathrm{O}_{3}$ concentration, the percentage of COPD hospitalization of residents with each $1 \mathrm{mg} / \mathrm{m}^{3}$ increase in CO enhanced by $0.05 \%, 0.08 \%, 0.09 \%, 0.24 \%, 0.05 \%$ and $0.18 \%$, respectively. Tian et al. (29) found that for every $90.8 \mu \mathrm{g} / \mathrm{m}^{3}$ increase in PM2.5, the number of outpatients and inpatients with COPD was enhanced by $2.38 \%$ (95\% CI: $2.22-2.53 \%$ ) and $6.03 \%$ (95\% CI: 5.19-6.87\%), respectively. The results of a national survey by Wang et al. (4) showed that the risk of COPD was 2.00 times (95\% CI: 1.36-2.92) with $\mathrm{PM} 2.5 \geq 75 \mu \mathrm{g} / \mathrm{m}^{3}$ than that with PM2.5 $<50 \mu \mathrm{g} / \mathrm{m}^{3}$. The risk of COPD with smoking and PM2.5 $\geq 75 \mu \mathrm{g} / \mathrm{m}^{3}$ was 4.92 times of that with non-smoking and PM2.5 $<50 \mu \mathrm{g} / \mathrm{m}^{3}$, indicating that there is an interaction between COPD risk and smoking. In summary, higher concentrations of air pollutants (PM10, $\mathrm{PM} 2.5, \mathrm{SO}_{2}, \mathrm{NO}_{2}, \mathrm{CO}$, etc.) may induce higher COPD morbidity, mortality and COPD exacerbations.

\section{Disease burden}

\section{Admission and readmission of COPD}

Recurrent acute exacerbation of chronic obstructive pulmonary disease (AECOPD) is an important event in the management of COPD. The most common causes of AECOPD are upper respiratory tract viral infections and tracheobronchial infections, increased bacterial load in the airways or the appearance of new strains in the airways. About $50 \%$ of AECOPD patients are complicated with upper respiratory tract viral infection, and $40 \%$ to $60 \%$ of AECOPD patients can isolate bacteria from sputum. In addition to viruses and bacteria, $3 \%$ to $5 \%$ of patients with AECOPD are caused by chlamydia pneumoniae, and AECOPD can also be caused by non-infectious factors, such as smoking, air pollution, and inhalation of allergens. The frequent reoccurrence of AECOPD accelerates disease progression, thereby increasing the hospitalization and readmission rate, increasing the medical burden, seriously affecting patient quality of life and increasing the risk of death. In 2007, 2.67-6.3\% of COPD patients were readmitted within 31 days in five large general hospitals in Beijing (30). The results of another analysis on COPD readmission rate between 2005 and 2015 in Dalian, Northeast China highlighted that the average readmission rate of COPD patients within a 30 -day period was $4.5 \%$, but there was no significant change in the admission rate over the study period (31). COPD readmission rates are much lower in China than in the UK $(13.3 \%$ to $23.63 \%)(32,33)$ and the US $(19.4 \%$ to $36.4 \%)(34,35)$. The influencing factors of readmission to hospital after discharge in COPD patients were closely related to COPD assessment test (CAT) score, comorbidities, acute treatment in the past year, emphysema index, depression score, and age $(36,37)$. The application of hospital-community-family linkage nursing model and self-management education (SEM) in patients with stable COPD helps to promote the recovery of pulmonary function, reduce dyspnea symptoms, reduce readmission rates, and improve quality of life $(38,39)$. When AECOPD patients are discharged from the hospital, it is necessary to clearly develop an effective long-term home maintenance therapy program for drugs, train the drug inhalation technique of patients, and provide education on the efficacy of maintenance therapy program for stable COPD (40).

\section{COPD mortality}

At present, COPD is the fourth leading cause of death both globally and in China (41). Further, by 2030 COPD is expected to represent the fifth highest disease burden and the third greatest global cause of death (42). Zhou et al. (43) showed that the age-standardized rate of COPD mortality in China decreased by 68.6\% from 1990 to 2017. The standardized mortality rate of COPD in China was $159.6 / 100,000$ in 1990, which decreased to $79.4 / 100,000$ by 2013 , a decrease of $50.3 \%$. The largest decrease, $70.2 \%$, was observed in Heilongjiang Province. The city with the highest increase in deaths from 1990 in 2013 was Chongqing ( $84.5 \%$ increase), and the city with the largest decrease was Tibet (42.9\% decrease) (44). In 2004, COPD was the third leading cause of death in urban areas and the second highest in rural areas of China, with COPD mortality rates of $67.30 / 100,000$ and $89.86 / 100,000$, respectively. The mortality rate decreased to $47.30 / 100,000$ and 66.24/100,000 in 2016 (fourth and third leading causes of death, respectively), with a higher mortality rate in rural areas than in urban areas. In 2016, the mortality rates of COPD were 12.86/100,000 and 394.37/100,000 in urban people aged 41 to 64 years old and those aged 65 years and older, respectively, and 18.61/100,000 and 524.51/100,000 in rural areas, respectively (45). Although the mortality rate of COPD in China has decreased, the mortality rate is still higher than the global average, and the number of deaths 
accounts for more than $30 \%$ of global deaths (46).

\section{Disability-adjusted life years (DALYs)}

Between 1990 and 2017, the age-standardized mortality rate of COPD decreased by $68.6 \%$ [ $95 \%$ uncertainty interval (UI): $62.0-70.9 \%$ ], and the age-standardized DALY of COPD decreased by $66.4 \%$ (95\% UI: 61.2-68.4\%). However, COPD was still the fourth leading cause of death. The DALY of COPD also increased from the fourth leading cause in 1990 to the third leading cause after ischemic heart disease and stroke in 2017 (13). The DALY of COPD among people aged 15 years and older in China decreased from 4,120.1/100,000 in 1990 to 1,575.9/100,000 in 2010 (43). Cui et al. (47) showed that the DALY caused by standardized COPD in the whole age group in China in 1990 was $2,558.30 / 100,000$, which decreased by $52.19 \%$ to $1,223.20 / 100,000$ in 2013. According to GDB data, DALYs caused by COPD in China decreased from 2,250.82/100,000 in 1990 to $1,445.53 / 100,000$ in 2017 , but were higher than those worldwide in the same year (1990: 1,263.93/100,000 to $2017: 1,038.02 / 100,000)(46)$. The above data indirectly reflect significant social, economic and medical progress made in China between 1990 and 2017. However, compared with the global average, the DALY rate caused by COPD in China is still high.

\section{Economic burden of COPD}

COPD causes a heavy economic burden to patients, their families and society. The annual per capita direct medical cost of COPD in China is between $\$ 72$ and $\$ 3,565$, accounting for $33.33 \%$ to $118.09 \%$ of the local average annual income (6). He et al. (48) investigated the economic burden of 723 patients with COPD in six Chinese cities (including Beijing) and found that the total direct cost was 13,314 yuan/year, accounting for about $40 \%$ of the total family income. Huang et al. (49) showed that the per capita direct economic burden of hospitalized patients with COPD in a tertiary care hospital in Beijing increased from $19,766.52$ yuan to $26,133.18$ yuan from 2005 to 2013 , with an average growth rate of $3.55 \%$. The per capita economic burden of patients with COPD in rural areas of Yunnan Province was $11,348.88$ yuan, which significantly exceeded the annual average family income of patients (50). In another study, 1,634 patients with COPD were followed up for 3 months in 83 tertiary hospitals in 17 provinces and cities of China. The results showed that the total economic burden of COPD was $10,617.65$ to $24,584.22$ yuan, of which the direct economic cost was $6,636.67$ to $15,402.86$ yuan respectively, therefore, the medical cost was more than 1.2 times the average income. Only $2.5 \%$ of the patients had 30-day medication cost lower than their daily salary (51). Overall, COPD causes a significant economic burden to patients and their families, and this can result in severe financial difficulties for many families.

\section{Comorbidities associated COPD}

The Global Initiative for Chronic Obstructive Lung Disease (GOLD) guidelines state that COPD also exhibits extrapulmonary comorbidities such as cardiovascular disease, metabolic disease, mental illness, and musculoskeletal system diseases. These comorbidities may contribute to the reduced quality of life, increased difficulty of treatment and increased risk of mortality in patients with COPD $(52,53)$. More than $70 \%$ of COPD patients had at least one comorbidity. For example, among 532 patients with stable COPD in Shanghai Respiratory Clinic, $73.9 \%$ had complications, where the top four most common complications were pulmonary hypertension (34.2\%), hypertension (27.1\%), bronchial asthma (25.4\%), and metabolic syndrome (22.9\%) (54). Another study showed that $89.2 \%$ of COPD patients had more than one concomitant disease, and the top five concomitant diseases were hypertension $(27.4 \%)$, lung cancer $(23.5 \%)$, cor pulmonale $(17.6 \%)$, hypoproteinemia $(11.9 \%)$ and liver disease (11.6\%) (55). These comorbidities increase the risk of COPD exacerbations, a decrease in quality of life, and decreasing survival in patients with COPD (56).

Overall, COPD often coexists with other diseases ultimately affecting the prognosis of COPD patients. These co-morbidities can be either independent of COPD, occur downstream of COPD or be dependent upon overlapping risk factors. Therefore, comprehensive assessment of COPD complications, alongside therapeutic intervention and management can reduce the risk of acute exacerbation and improve the prognosis of COPD patients.

\section{The understanding and treatment of COPD in China}

\section{Public awareness of COPD}

Currently, the rate of awareness for COPD and pulmonary function tests among Chinese residents is low. The 
diagnostic yield of COPD patients in China ranges from $23.61 \%$ to $30.00 \%$, with an admission rate between $8.78 \%$ and $35.60 \%$, where an additional $~ 50 \%$ of patients diagnosed with COPD received outpatient treatment (6). A survey of the Chinese Adult Pulmonary Health Study showed that $60 \%$ of COPD patients had no subjective symptoms and $39.8 \%$ reported typical symptoms of COPD. Of the patients diagnosed, only $2.6 \%$ were aware of having COPD and $12 \%$ underwent pulmonary function tests (5). Among the 3,000 permanent residents who were aged $\geq 40$ years and were from five COPD monitoring sites in Yunnan Province, $1.8 \%$ had received pulmonary function tests (57) In Fujian Province, only $6.1 \%$ of those surveyed had heard of COPD, $2.6 \%$ of people were aware of pulmonary function tests and $4.3 \%$ were aware of COPD symptoms and risk factors (58). A survey of people aged 40 years and above in 31,125 monitoring sites across China showed that $9.2 \%$ of people were aware of COPD, $3.6 \%$ of people were aware of pulmonary function tests and $5.8 \%$ of people had COPD-related knowledge (59). Eighty-nine-pointfive percent of COPD patients had at least one risk factor, which increased with age (60).

COPD can be prevented and controlled. Therefore, attention should be paid to the early prevention of COPD. Extensive publicity and education and effective community management should be carried out to improve the awareness of community residents and patients, thus effectively reducing the incidence of COPD, delaying disease progression and improving the quality of life of patients.

\section{Awareness of COPD amongst doctors}

A large proportion of primary care physicians in China lack knowledge about COPD. Around 59.0\% of physicians from tertiary hospitals in Northeast China, which include respiratory specialists, correctly believe that a pulmonary function test is an important basis for the diagnosis of COPD (61). A survey on the awareness of COPD was conducted in general practitioners from four primary health service centers in Shanghai. This survey found that 55.15\% of general practitioners were aware of the gold standard for the COPD diagnosis, $56.97 \%$ were aware of the main clinical manifestations of COPD, 33.94\% were aware of the grading standard of COPD and finally only $44.74 \%$ knew the correct pharmacological treatment for COPD (62). Another survey on COPD knowledge in 325 doctors from the community of Pudong New District, Shanghai reported that whilst $60.2 \%$ of the doctors received the training on the relevant knowledge of COPD, only $21.7 \%$ of the doctors knew the main content of the guidelines for the diagnosis and treatment of COPD and $49.7 \%$ of the doctors believed that they could not provide community health care services for COPD (63). Additionally, a survey on the relevant knowledge and standardized treatment of COPD was conducted with physicians from one secondary hospital and eight communities in Chongming County, Shanghai. The study highlighted that $20.8 \%$ of surveyed physicians knew the definition of COPD, only $11.3 \%$ were aware of the diagnostic criteria for COPD and $96.2 \%$ of the respondents did not know the relevant contents of the Guidelines for the Diagnosis and Treatment of Chronic Obstructive Pulmonary Disease (2007 Revision) in China (64). In terms of standardized treatment, $65.1 \%$ of doctors overused antibiotics without indications, a further $10.4 \%$ of the doctors prescribed inhaled $\beta 2$-agonists for patients exhibiting wheezing and none of the doctors prescribed inhaled anticholinergic drugs (65).

At present, most local doctors have limited basic knowledge and a lack of the required clinical knowledge and skills for COPD diagnosis and treatment. Additionally, most local medical and health institutions do not have the equipment for pulmonary function tests. Meanwhile, local doctors have a limited awareness of performing pulmonary function tests and are typically unskilled at performing them, resulting in high misdiagnosis and missed diagnosis of COPD. Therefore, actively promoting primary pulmonary function tests can effectively reduce the rate of misdiagnosis and missed diagnosis and contribute to the early detection and treatment of COPD. At the same time, it is urgent to strengthen the education and training of grassroots doctors on the knowledge and skills of COPD and improve the standardized prevention and treatment ability of grassroots doctors for COPD.

\section{Current status of COPD treatment}

In recent years, Chinese respiratory experts have successively introduced multiple COPD diagnosis and treatment guidelines to support doctors in medical institutions at all-levels to standardize the treatment of COPD. These guidelines are in close alignment with GOLD guidelines and in accordance with the current situation in China.

However, currently treatment for COPD in China is not optimal due to the vast geographical area and 
uneven population distribution, economic development and education, alongside regional differences in the management of chronic diseases, and the insufficient understanding of COPD in a proportion of doctors. In 2010, a survey and study on the treatment status and selfknowledge of multicenter patients in 11 large hospitals in China showed that most COPD patients had an insufficient understanding of the disease (66), and $59.1 \%$ of patients believed that the treatment goal of COPD was to reduce symptoms. In the past 3 months, $39.8 \%, 21.7 \%$ and $53.7 \%$ of COPD patients were treated with isopropyl bromide, tiotropium bromide and theophylline sustained-release tablets respectively, where $72 \%$ of COPD patients took the prescribed medication according to their doctor's advice. More than $80 \%$ of these patients did not know the adverse reactions of commonly used inhaled drugs, $42 \%$ of these patients often took antibiotics, $70.5 \%$ of the patients did not receive respiratory rehabilitation guidance and $77.4 \%$ of the patients believed that COPD required long-term regular treatment. The study results of Yuan et al. (67) showed that COPD patients had a serious lack of awareness of the disease and non-standard treatment in the stable phase. Four out of 5 patients themselves do understand COPD, and most COPD patients had visited primary hospitals, which have limited medical conditions and a relative lack of equipment and personnel, resulting in ineffective disease control. $60 \%$ of COPD patients did not know that patients required home oxygen therapy, $73.0 \%$ did not know that oxygen inhalation required low-flow, $66 \%$ did not know the concept of pulmonary rehabilitation, and $82.4 \%$ did not know the relevant content of pulmonary rehabilitation. An analysis of frequently prescribed medications for COPD in primary hospitals (68) showed that theophylline $(81.2 \%)$ was the most used prescription for the treatment of mild to moderate COPD, followed by expectorant drugs $(79.7 \%)$, and the proportion of patients receiving inhalation therapy did not exceed $60 \%$. For patients with severe COPD, the most used prescription was expectorants (82.6\%); followed by theophyllines (79.7\%), and inhaled corticosteroids (73.9\%). The survey results of medication in elderly patients with COPD showed that the highest medication was cough expectorant $(94.1 \%)$, followed by phosphodiesterase inhibitors (90.2\%), glucocorticoids and muscarinic cholinergic receptor antagonists accounted for only $9.8 \%$. The survey for compliance with medication showed that $69.2 \%$ of patients used drugs according to the doctor's advice during disease exacerbation and stopped the drug spontaneously after the condition was stable and
$32.4 \%$ followed the advice of surrounding patients when choosing drug treatment. Twenty-five-point-five percent chose to buy drugs by themselves in pharmacies, and $8.9 \%$ used health-care products (69). These seriously affect the treatment of patients with stable COPD, which may lead to disease progression of COPD and increased mortality.

\section{Prevention and treatment strategy of COPD in China}

Based on the aforementioned domestic research data, a series of prevention and treatment strategies have been introduced. These are summarized here at the level of national policy, society and scientific research.

\section{National policy level}

Prevention and control of COPD have been included in the national series of policies for the prevention and control of chronic diseases. In 2012, COPD was first included in the National Work Plan for the Prevention and Treatment of Chronic Diseases (70). In 2014, it was first included in the National Chronic Obstructive Pulmonary Disease Surveillance Project-Monitoring Project for Chinese Residents, covering permanent residents aged 40 years and above in 125 monitoring counties (districts) in 31 provinces in China, and an on-site survey was carried out every 5 years (71). In 2015, it was first included in the National Work Plan for the Prevention and Treatment of Chronic Diseases (72). In 2017, it was included in the tiered diagnosis and treatment project, and meanwhile the technical plan for tiered diagnosis of COPD was formulated (73). In the Medium- and Long-Term Plan for the Prevention and Treatment of Chronic Diseases in China (2017-2025) issued in 2017, it is proposed to include pulmonary function tests in the routine physical examination for patients aged 40 years old and above, reaching $15 \%$ by 2020 and $25 \%$ by 2025 (74). It is pointed out in the Action for Healthy China (2019-2030) in 2019 that by 2022 and 2030, the mortality rate of chronic respiratory diseases in the population aged 70 years or younger are expected to decrease to $9 / 100,000$ and 8.1/100,000, respectively; and, the awareness rate of COPD in the residents aged 40 years and older is expected to reach more than $15 \%$ and $30 \%$, respectively. In people aged 40 and over with chronic respiratory diseases pulmonary function tests should be performed annually (75). Meanwhile, the understanding of COPD and awareness of the preventative measures and treatment of COPD by local 
medical staff, patients and residents should be strengthened. The implementation of the above policies will effectively promote the prevention and control of COPD in China.

\section{At the society/society level}

The respiratory disease prevention and control system in China is currently trailing behind other developed nations. The development of respiratory science remains unbalanced, which limits preventative and control measures, this is especially important given the increasingly severe morbidity in China.

To solve this problem, the Chinese Thoracic Society (CTS) and the Chinese Association of Chest Physicians (CACP) jointly promote action for the prevention and control of chronic respiratory diseases and propose initiatives for colleagues in the respiratory community as well as wider Chinese society. Of these 15 special initiatives, eight are related to respiratory health, including health knowledge popularization, national fitness, tobacco control, health environment promotion, occupational health protection, cancer prevention and control, chronic respiratory disease prevention and control, and infectious and endemic disease prevention and control.

Additionally, a network of standardized prevention and treatment systems for respiratory diseases in local medical institutions and standardized construction of PCCM (Department of Pulmonary and Critical Care Medicine) was established. A grassroots respiratory disease prevention and treatment alliance was established to promote the training and education of local doctors, thereby aiming to improve the level of standardized diagnosis and treatment of diseases, to promote the development of respiratory disciplines and improve the level of specialist diagnosis and treatment. In August 2016, a national respiratory specialist medical alliance led by China-Japan Friendship Hospital was established to realize cross-provincial tiered diagnosis and treatment specialties, implement two-way referral and tiered diagnosis and treatment, cultivate talent, carryout clinical research, enable patients to obtain scientific, convenient, economical, efficient and continuous medical care, and promote the development of the discipline (76).

\section{Scientific research level}

Amongst the "Thirteenth Five-Year Plan" series of special projects issued by the Ministry of Science and Technology, multiple special projects involve COPD, including the precision medicine special project, the major chronic disease special project and the air pollution special project. Together these provide opportunities and challenges for the in-depth study of the etiology, pathogenesis, clinical phenotype, complications, combined drug therapy, diagnosis and treatment of acute exacerbation of respiratory pathologies, treatment of respiratory failure and excellent control of COPD.

Additionally, the "Special Project for Clinical Research of Chronic Obstructive Pulmonary Disease" established by the Public Welfare Fund for Clinical Research of Respiratory Diseases, Song Qingling Foundation, China, has provided funding to carry-out clinical study of COPD. The Mingdao Respiratory School Chronic Obstructive Pulmonary College will also be established to cultivate the training of COPD specialists, and this will promote the remote consultation for chronic respiratory diseases. These initiatives will promote the standardized diagnosis and treatment of COPD in China and benefit the prevention and treatment of COPD.

\section{Limitations}

It is extremely difficult to conduct large-scale epidemiological survey of COPD in China, due to its vast territory, highly mobile large population and regional differences in wealth and education. Therefore, in recent years, there has been limited large-scale epidemiological studies of COPD and the data discussed here were most recently collected in 2017, with limited data from the past 3 years. It is gratifying that, during the writing of this manuscript, the "Training Course on Early Screening Intervention Capacity Improvement Project for Respiratory Diseases" led by the National Health and Family Planning Commission of China is being carried out nationwide to further standardize and guide the diagnosis and treatment of respiratory diseases and standardize the operating process of pulmonary function tests in primary hospitals. At the same time, the state has invested hundreds of millions of yuan to provide primary hospitals with spirometers to actively promote pulmonary function screening and standardize their operating techniques. It is believed that in the near future we will conduct additional comprehensive studies, which will also provide a stronger basis for the formulation of national policies. Whilst we have provided a thorough review of the literature in this review, we acknowledge that we have not used a systematic literature search. 


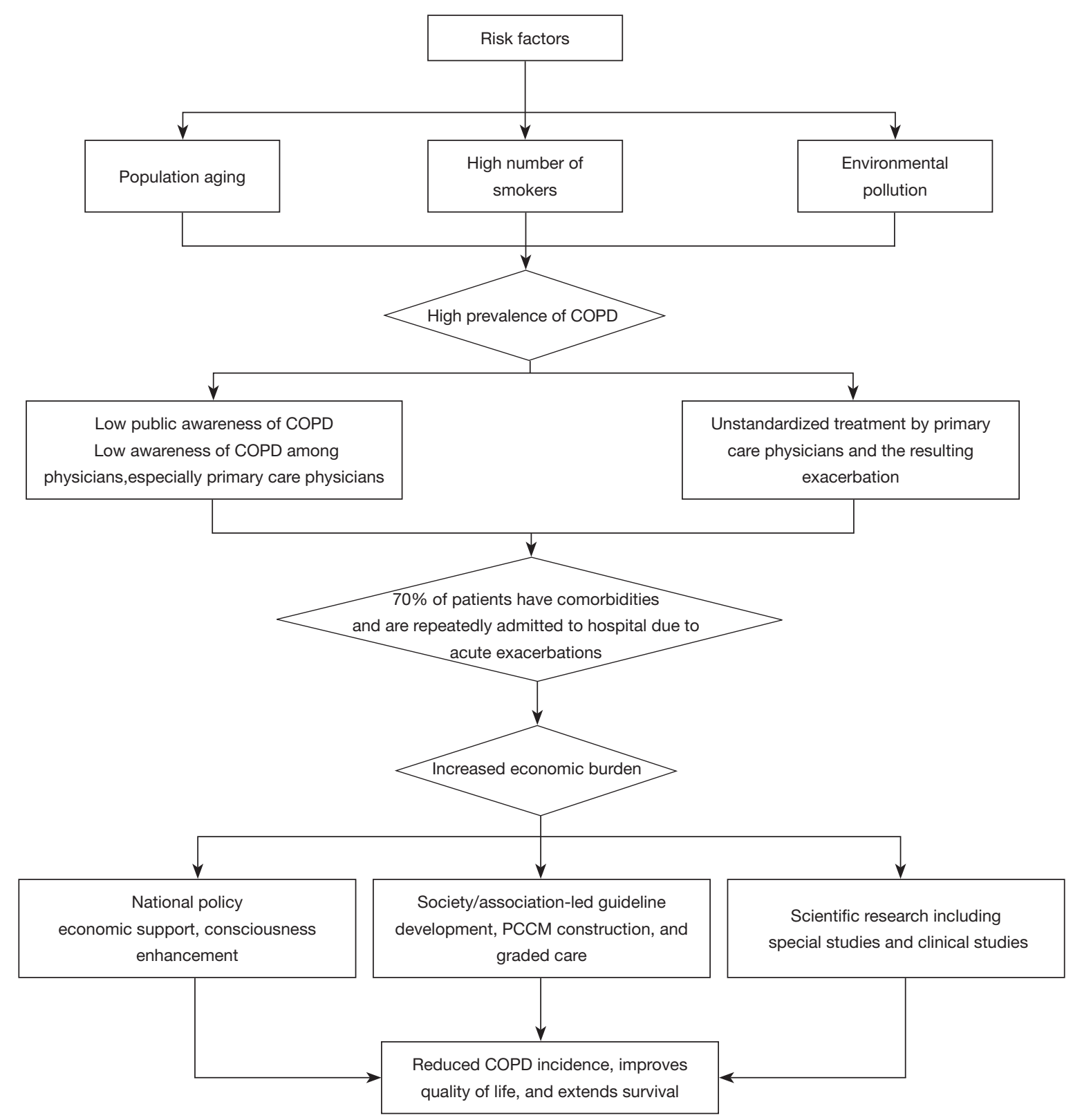

Figure 1 Current status and preventive strategies of chronic obstructive pulmonary disease (COPD) in China.

\section{Summary}

The current status of COPD in China is summarized by Figure 1. COPD is one of the important public health problems both in China and globally. It is of great importance to actively control the relevant risk factors, effectively prevent its occurrence and development, to improve its prognosis and the quality of life of COPD patients. We should not only improve patients' and their families' understanding of the disease (such as smoking cessation and reducing secondhand smoke exposure), but also the diagnosis and treatment level of medical professionals. Social support for pollution reduction and prevention, as well as national policy support for COPD are also required. Only through a multi-faceted cooperation and a joint effort can we effectively promote the establishment and improvement of COPD prevention strategies and systems in China. 


\section{Acknowledgments}

Funding: This work was supported by National Natural Science Foundation of China (No. 81660003, 81860729, 81970018).

\section{Footnote}

Provenance and Peer Review: This article was commissioned by the Guest Editor (Ki-Suck Jung and Chin Kook Rhee) for the series "current epidemiology and policies of COPD worldwide" published in Fournal of Thoracic Disease. The article has undergone external peer review.

Reporting Checklist: The authors have completed the Narrative Review reporting checklist. Available at http:// dx.doi.org/10.21037/jtd-20-2051

Conflicts of Interest: All authors have completed the ICMJE uniform disclosure form (available at http:// dx.doi.org/10.21037/jtd-20-2051). The series "current epidemiology and policies of COPD worldwide" was commissioned by the editorial office without any funding or sponsorship. The authors have no other conflicts of interest to declare.

Ethical Statement: The authors are accountable for all aspects of the work in ensuring that questions related to the accuracy or integrity of any part of the work are appropriately investigated and resolved.

Open Access Statement: This is an Open Access article distributed in accordance with the Creative Commons Attribution-NonCommercial-NoDerivs 4.0 International License (CC BY-NC-ND 4.0), which permits the noncommercial replication and distribution of the article with the strict proviso that no changes or edits are made and the original work is properly cited (including links to both the formal publication through the relevant DOI and the license). See: https://creativecommons.org/licenses/by-nc-nd/4.0/.

\section{References}

1. Chan KY, Li X, Chen W, et al. Prevalence of chronic obstructive pulmonary disease (COPD) in China in 1990 and 2010. J Glob Health 2017;7:020704.

2. Cheng X, Li J, Zhang Z. Analysis of basic data of the study on prevention and treatment of COPD and chronic cor pulmonale. Zhonghua Jie He He Hu Xi Za Zhi 1998;21:749-52.

3. Zhong N, Wang C, Yao W, et al. Prevalence of chronic obstructive pulmonary disease in China: a large, Population- based survey. Am J Resp Crit Care Med 2007;176:753-60.

4. Wang C, Xu J, Yang L, et al. Prevalence and risk factors of chronic obstructive pulmonary disease in China (the China Pulmonary Health $[\mathrm{CPH}]$ study): a national cross-sectional study. Lancet 2018;391:1706-17.

5. Fang L, Gao P, Bao H, et al. Chronic obstructive pulmonary disease in China: a nation wide prevalence study. Lancet Respir Med 2018;6:421-30.

6. Zhu B, Wang Y, Ming J, et al. Disease burden of COPD in China: a systematic review. Int J Chron Obstruct Pulmon Dis 2018;13:1353-64.

7. Shi W, Liu Y, Zhang F, et al. Survey on the prevalence and risk factors of chronic obstructive pulmonary disease in the population over 40 years in parts of areas of Hebei province. Hebei Medical Journal 2018;40:1419-22.

8. Chen Z, Peto R, Zhou M, et al. Contrasting male and female trends in tobacco-attributed mortality in China: evidence from successive nationwide prospective cohort studies. Lancet 2015;386:1447-56.

9. Ding $\mathrm{Y}, \mathrm{Xu} \mathrm{J}$, Yao J, et al. The analyses of risk factors for COPD in the Li ethnic group in Hainan, People's Republic of China. Int J Chron Obstruct Pulmon Dis 2015;10:2593-600.

10. He Y, Jiang B, Li LS, et al. Secondhand smoke exposure predicted COPD and other tobacco-related mortality in a 17-year cohort study in China. Chest 2012;142:909-18.

11. Yin $\mathrm{P}$, Jiang CQ, Cheng KK, et al. Passive smoking exposure and risk of COPD among adults in China: the Guangzhou Biobank Cohort Study. Lancet 2007;370:751-7.

12. Chinese Center for Disease Control and Prevention. Report on chronic disease risk factor surveillance in China 2010. Beijing: People's Medical Publishing House, 2012.

13. Chinese Center for Disease Control and Prevention. 2010 Global Adult Tobacco Survey: China Report. Beijing: China Three Gorges Press, 2011.

14. Wang N, Feng YJ, Bao HL, et al. Survey of smoking prevalence in adults aged 40 years and older in China, 2014. Zhonghua Liu Xing Bing Xue Za Zhi 2018;39:551-6.

15. Cong S, Feng YJ, Bao HL, et al. Analysis on passive smoking exposure in adults aged 40 years and older in China, 2014. Zhonghua Liu Xing Bing Xue Za Zhi 2018;39:557-62.

16. Liu S, Zhou Y, Wang X, et al. Biomass fuels are the 
probable risk factor for chronic obstructive pulmonary disease in rural South China. Thorax 2007;62:889-97.

17. Lin C, Zhai X, Deng T. Meta analysis on risk factors related to chronic obstructive Lung disease in Chinese population. Pract Prev Med 2018;25:191-4.

18. An J, Bao HL, Fang LW. Relationship between biomass smoke exposure and chronic obstructive pulmonary disease among residents in China: a meta-analysis. Chinese Journal of Public Health 2016;32:999-1004.

19. Feng YJ, Fan J, Cong S, et al. Current status of household polluting fuel use in adults aged 40 years and older in China, 2014. Zhonghua Liu Xing Bing Xue Za Zhi 2018;39:569-73.

20. He YZ, Bao HL, Feng YJ, et al. Prevalence of biomass fuel exposure in women aged 40 years and older in China, 2014. Zhonghua Liu Xing Bing Xue Za Zhi 2018;39:574-9.

21. Wang BH, Cong S, Bao HL, et al. Analysis on occupational exposure to dust and harmful gas and corresponding protection in adults aged 40 years and older in China, 2014. Zhonghua Liu Xing Bing Xue Za Zhi 2018;39:563-8.

22. Zhou Y, Wang C, Yao W, et al. Occupational Exposure to Dusts/Gases/Fumes is Contributed to Chronic Obstructive Pulmonary Disease and Respiratory Symptoms. Chinese Journal of Respiratory and Critical Care Medicine 2009;8:6-11.

23. Liao $\mathrm{P}$, Zhe $W$, Liu L, et al. Analysis of the morbidity and influencing factors of chronic obstructive pulmonary disease in residents ( $\geq 40$ years old) of Xinjiang monitoring stations. Chinese Journal of Prevention and Control of Chronic Diseases 2018;26:576-9.

24. Qian Y, Han M, Jin W, et al. Association between Ambient Air Pollution and COPD Mortality in Shanghai. J Environ Occup Med 2015,32:1093-7.

25. Wu XY, Li GX, Wang XY, et al. Correlation study between respiratory death and airborne particles in Beijing: Spatiotemporal analysis based on satellite remote sensing data. Beijing Da Xue Xue Bao Yi Xue Ban 2017;49:409-17.

26. Jin Y, Cheng Y, Wang H, et al. Effects of air pollution from burning coal on respiratory diseases in adults. Wei Sheng Yan Jiu 2001;30:241-3, 246.

27. Jin Y, He G, Liu F, et al. Quantified study on human health impact caused by coal-burning air pollution in China. Wei Sheng Yan Jiu.2002;31:342-8.

28. Li C, Zhou L, Wang S, et al. The effect of air pollution on hospitalization of chronic obstructive pulmonary disease: a meta-analysis. Chinese Journal of Gerontology 2016;36:1141-5.
29. Tian Y, Xiang X, Juan J, et al. Short-term effects of ambient fine particulate matter pollution on hospital visits for chronic obstructive pulmonary disease in Beijing, China. Environ Health 2018;17:21.

30. Chen H, Jiao Y, Zhao M, et al. A Comparative Analysis of "Re-entry Category" Indicators in Five Major General Hospitals in Beijing. Chinese Health Quality Management 2010;17:4-7.

31. Liu H, Wang N, Chen W, et al. Hospitalization Trends in Adult Patients with COPD and Other Respiratory Diseases in Northeast China from 2005 to 2015. Biomed Res Int 2018;2018:1060497.

32. Morton K, MacNeill S, Sanderson E, et al. Evaluation of 'care bundles' for patients with chronic obstructive pulmonary disease (COPD): a multisite study in the UK. BMJ Open Respir Res 2019;6:e000425.

33. McGill C. Reducing COPD Rehospitalizations. Home Healthc Now 2020;38:80-5.

34. Gershon AS, Thiruchelvam D, Aaron S, et al. Socioeconomic status (SES) and 30-day hospital readmissions for chronic obstructive pulmonary (COPD) disease: A population-based cohort study. PLoS One 2019;14:e0216741.

35. Zhou Y, Rong R, Huang Y, et al. Influencing Factors and Nomogram Model for Readmission in AECOPD Patients within 30 Days after Discharge. Practical Journal of Cardiac Cerebral Pneumal and Vascular Disease 2020;28:57-63.

36. Wei X, Ma Z, Yu N, et al. Risk factors predict frequent hospitalization in patients with acute exacerbation of COPD. Int J Chron Obstruct Pulmon Dis 2017;13:121-9.

37. Zhou X, Zhao C, Huang Z, et al. Relevant analysis of hospitalized COPD patients complicated with depression and their re-acute exacerbation and hospitalization. Journal of Clinical Pulmonary Medicine 2019;24:1767-71.

38. Suo Z. Application of hospital-community-family linkage nursing mode in patients with stable COPD. Journal of Diseases Monitor \& Control 2021;15:69-71, 76.

39. Wang T, Tan JY, Xiao LD, et al. Effectiveness of diseasespecific self-management education on health outcomes in patients with chronic obstructive pulmonary disease: An updated systematic review and meta-analysis. Patient Educ Couns 2017;100:1432-46.

40. Cai B, Chen R. Chinese expert consensus on diagnosis and treatment of acute exacerbation of chronic obstructive pulmonary disease (AECOPD) (updated in 2017). International Journal of Respiration 2017.37:1041-57.

41. Global Burden of Disease Study 2013 Collaborators. 
Global, regional, and national incidence, prevalence, and years lived with disability for 301 acute and chronic diseases and injuries in 188 countries, 1990-2013: a systematic analysis for the Global Burden of Disease Study 2013. Lancet 2015;386:743-800.

42. Luo W, Lin Y. Mortality time trends and Projection of chronic obstructive pulmonary disease in Xiamen. Journal of Diseases Monitor \& Control 2016;10:783-5.

43. Zhou M, Wang H, Zeng X, et al. Mortality, morbidity, and risk factors in China and its provinces, 1990-2017: a systematic analysis for the Global Burden of Disease Study 2017. Lancet 2019;394:1145-58.

44. Yin P, Wang H, Vos T, et al. subnational analysis for mortality and prevalence of chronic obstructive pulmonary disease in China 1990- 2013: Findings from Global Burden of Disease Study (GBD)2013. Chest 2016;150:1269-80.

45. Zhu J. Analysis on the Death Patterns and Death levels between Urban and Rural Population in China from 2004 to 2016. Master's thesis. Zhengzhou: Zhengzhou University, 2018.

46. GBD Results Tool. Available online: http://ghdx. healthdata.org/gbd-results-tool

47. Cui F, Yu C, Nie D, et al. Analysis of the trend of disability-adjusted life year and health-adjusted life expectancy in China between 1990 and 2013. Modern Preventive Medicine 2016;43:2959-2962, 2996.

48. He QY, Zhou X, Xie CM, et al. Impact of chronic obstructive pulmonary disease on quality of life and economic burden in Chinese urban areas. Zhonghua Jie He He Hu Xi Za Zhi 2009;32:253-7.

49. Huang J. Analysis on Direct Economic Burden of Hospitalized Patients with COPD and Its Influencing Factors in a Three-level Hospital in Beijing. Medicine and Society 2015;28:19-22.

50. Mao H, Cai L, Shu Z, et al. Analysis of Prevalence and Economic Burden of Chronic Obstructive Pulmonary Disease in Rural Residents of Yunnan Province. Chinese Journal of Prevention and Control of Chronic Diseases 2012:20:244-6.

51. Li J, Feng R, Cui Y, et al. Analysis on the Affordability and Economic Risk for Using Medicine to Treat Patients with Chronic Obstructive Pulmonary Disease in Tier 3 Hospitals in China. Chinese Health Economics 2015;34:66-8.

52. Motegi T, Jones RC, Ishii T, et al. A comparison of three multidimensional indices of COPD severity as predictors of future exacerbations. Int J Chron Obstruct Pulmon Dis 2013;8:259-71.

53. Mannino DM, Thorn D, Swensen A, et al. Prevalence and outcomes of diabetes, hypertension and cardiovascular disease in COPD. Eur Respir J 2008;32:962-9.

54. Feng X, Ge H, Gu W, et al. Characteristics and clinical significance of major comorbidities of stable COPD in some Tier2 and Tier3 hospitals in Shanghai, China. Acad J Sec Mil Med Univ 2021;42:177-88.

55. Chen HP, Hu MD, Zhang JG, et al. Complications of chronic obstructive pulmonary disease and their effects on prognosis. Med J Chin PLA 2020;45:834-9.

56. Smith MC, Wrobel JP. Epidemiology and clinical impact of major comorbidities in patients with COPD. Int J Chron Obstruct Pulmon Dis 2014;9:871-88.

57. Shao Y, Yang Y, Qin Ming, et al. Analysis of Influencing factors of pulmonary function examination in Yunnan Province. Chinese Journal of Prevention and Control of Chronic Diseases 2019;27:739-43.

58. Chen J, Lin X, Zhang Y, et al. Analysis of Cognitive Status of Chronic Obstructive Nondisease among Residents Aged 40 Years and Older in Fujian Province. Chronic Pathematology Journal 2019;20:347-50.

59. Fan J, Wang N, Fang L, et al. Awareness of knowledge about chronic obstructive pulmonary disease and related factors in residents aged 40 years and older in China, 2014. Chinese Journal of Epidemiology 2018;39:586-92.

60. Bao HL, Cong S, Wang N, et al. Survey and analyses of population at high risk of chronic obstructive pulmonary disease in China, 2014. Zhonghua Liu Xing Bing Xue Za Zhi 2018;39:580-5.

61. Wu C Zhou X, Zhao L, et al. Cross-sectional survey of the congition level of physicians from tertiary hospitals in Northeast China in the treatment of chronic obstructive pulmonary disease. Practical Pharmacy and Clinical Remedies 2016;19:568-72.

62. Li X. Epidemiological study of COPD area in Shanghai and recognition of COPD general practitioners. Master's thesis. Shanghai: Fudan University, 2012.

63. Qiu H, Ruan X, Zhou X, et al. Cognition survey on chronic obstructive pulmonary disease among community doctors in Pudong New Area in Shanghai. Shanghai Journal of Preventive Medicine 2018;30:750-4.

64. Group of Chronic Obstructive Pulmonary Diseases, Branch of Respiratory Diseases, Chinese Medical Association. Guidelines for diagnosis and treatment of chronic obstructive pulmonary disease (revised 2007). Chinese Journal of Tuberculosis and Respiratory Diseases 2007;30:8-17.

65. Zhou E, Luo Y, Xu W, et al. Standardized treatment and management of COPD (a questionaire by local physicians 
in Shanghai suburban areas). Clinical Misdiagnosis and Mistherapy 2010;23:9-11.

66. Chen YH, Yao WZ, Kang J, et al. Attitudes and actions of chronic obstructive pulmonary disease patients on treatment:a national multi-center investigative study. Zhonghua Jie He He Hu Xi Za Zhi 2010;33:750-3.

67. Qi M, Han Q, Jia R, et al. Status of disease awareness and treatment in patients with chronic obstructive pulmonary disease in Shandong province. J Prev Med Chin PLA 2012;35:656-7.

68. Yang Y. Analysis of treatment status of chronic obstructive pulmonary disease by physicians in township hospitals. China Rural Health 2015;9:62-3.

69. Tong C, Du Y, Liu X. Investigation on cognition and treatment of elderly patients with chronic obstructive pulmonary disease at stable stage in Taiyuan community. Chinese Journal of Geriatrics Research (Electronic Edition) 2012;2:25-7.

70. Notice of "China Chronic Disease Prevention Work Plan (2012-2015)" [EB / OL]. (2012-05-021)[2020-01-15]. Available online: http://www.nhc.gov.cn/wjw/gfxwj/201304/ b8de7b7415ca4996b3567e5a09e43300.shtml

71. Notice of the General Office of the National Health and Family Planning Commission on Chinese Residents'

Cite this article as: Quan Z, Yan G, Wang Z, Li Y, Zhang J, Yang T, Piao H. Current status and preventive strategies of chronic obstructive pulmonary disease in China: a literature review. J Thorac Dis 2021;13(6):3865-3877. doi: 10.21037/jtd-202051
Chronic Diseases and Nutrition Monitoring Work Program (Trial) [EB / OL]. (2014-10-14) [2020-01-15]. Available online: http://www.nhc.gov.cn/jkj/s5878/201409/ 9b0f5f9e50a9457fb54f140c6208997b.shtml

72. State Council. "Thirteenth Five-Year Plan" Health and Wellness Plan [EB / OL]. (2016-12-27)[2020-01015]. Available online: http://www.gov.cn/zhengce/ content/2017-01/10/content_5158488.htm

73. Notice on the technical plan for the diagnosis and treatment of chronic obstructive pulmonary disease [EB / OL]. (2017-02-09) [2020-01-15]. Available online: http:// www.ncis.cn/page.jsp? $i d=146 \& \bmod =100$

74. The General Office of the State Council. China's Medium and Long-Term Plan for the Prevention and Treatment of Chronic Diseases (2017-2025) [EB / OL]. (2017-0122) [2017-11-20]. Available online: http://www.gov.cn/ zhengce/content/2017-02/14/content_5167886.htm

75. Healthy China Action Committee. Healthy China Action (2019-2030) (2019-07-15) [2020-01-15]. Available online: http://www.gov.cn/xinwen/2019-07/15/ content_5409694.htm

76. Jia C, Li S, Zhang C, et al. Development and practice of medical alliances for respiratory diseases in China. Chinese Journal of Hospital Administration 2019;35:617-9. 